\title{
Computational modeling of atrial fibrillation
}

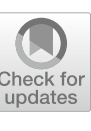

\author{
Oh-Seok Kwon, Inseok Hwang and Hui-Nam Pak ${ }^{*}$ (1)
}

\begin{abstract}
With the aging society, the prevalence of atrial fibrillation (AF) continues to increase. Nevertheless, there are still limitations in antiarrhythmic drugs (AAD) or catheter interventions for AF. If it is possible to predict the outcome of AF management according to various AADs or ablation lesion sets through computational modeling, it will be of great clinical help. AF computational modeling has been utilized for in-silico arrhythmia research and enabled high-density entire chamber mapping, reproducible condition control, virtual intervention, not possible clinically or experimentally, in-depth mechanistic research. With the recent development of computer science and technology, more sophisticated and faster computational modeling has become available for clinical application. In particular, it can be applied to determine the extra-PV target of persistent AF catheter ablation or to select the AAD with the best effect. AF computational modeling combined with artificial intelligence is expected to contribute to precision medicine for more diverse uses in the future. Therefore, in this review, we will deal with the history, development, and various applications of computation modeling.
\end{abstract}

Keywords: Atrial fibrillation, Computational modeling, Ablation, Antiarrhythmic drug

\section{Introduction}

Atrial fibrillation (AF) is a chronic, progressive arrhythmia that accounts for $20-25 \%$ of the causes of ischemic stroke, increases the risk of dementia by 2.5 times, and accompanies $30 \%$ of heart failures. As of 2016 , the prevalence of AF in Korea reached 1.6\%, but that figure continues to increase due to the aging population, improved survival of patients with heart disease, and environmental pollution [1]. The prevalence of AF is expected to be 3.5\% in 2030 when the population begins to decline in Korea, but the prevalence of AF is expected to increase continuously. Since AF is asymptomatic in over $40 \%$ of cases, the diagnosis of subclinical paroxysmal AF is quite difficult [2]. However, subclinical AF also increases the risk of stroke by 2.5 times if it lasts more than six minutes [3]. In spite of multifactorial conditions that affect the development of AF, it is a heritable disease, especially in the case of AF occurring at a young age [4]. The effectiveness of antiarrhythmic drugs (AADs), the first-line treatment

*Correspondence: hnpak@yuhs.ac

Division of Cardiology, Department of Internal Medicine, Yonsei University

Health System, 50-1 Yonsei-ro, Seodaemun-gu, Seoul, Republic of Korea for AF rhythm control, is also affected by this heritability [5]. Drug therapy for AF has limitations due to individual differences in antiarrhythmic effects and drug toxicity [6, 7]. Paradoxically, due to the limitations of drugs, catheter ablation or AF surgery has been developed, and the superior effect of non-pharmacological treatment of AF over AAD treatment has been demonstrated $[8,9]$. Currently, AF catheter ablation (AFCA) has become a common treatment for AAD-resistant AF patients, and it reduces heart failure mortality [10], overall AF mortality and hospitalization rates [11], risks of stroke [12] and dementia [13], and improves renal function [14]. However, AFCA is not a perfect procedure; while its one-year success rate is $75-90 \%$, it shows continuous recurrence and its five-year recurrence rate reaches $40-50 \%$ [15]. With the development of intelligent technology and the improvement of computational power, computational modeling is now being tried in various ways in clinical diagnosis and treatment. This review intends to examine the development and application of AF computational modeling to overcome the limitations of pharmacological and catheter ablation treatments for AF. 


\section{History, evolution, and hurdles of AF computational modeling}

AF computational modeling has been used in various basic electrophysiological studies since Moe et al. reported on a human atrial cellular model in 1964 [16, 17]. With the development of experimental cellular electrophysiology, AF modeling of the original prototype has been continuously upgraded, and various computational models with more accurate human atrial cellular ion channel properties have been developed and applied (Fig. 1). The Courtemanche model and the Nygren model were developed with applications of rate-dependent action potential change, restitution, and calcium dynamics $[18,19]$. These have slightly different formulas of ion currents, pumps, or exchangers, resulting in divergent behavior in rate-dependent $\mathrm{AP}$ and restitution of AP duration [20]. Later, the Courtemanche model was upgraded to the Krummen model [21] and the Nygren model was advanced to the Maleckar [22] and Koivumaki [23] models. In addition, according to the AF burden, Wilhelms proposed a chronic AF model [20], and Voigt developed a paroxysmal AF model [24]. Various AF modelings applying atrial tissue anatomy and histology have been applied, and sophisticated realistic atrial computation modeling that reflects the location of the myocardial scar has been introduced by applying late-gadolinium enhancement of cardiac magnetic resonance imaging (MRI) [25, 26].

Nevertheless, clinical studies using AF computational modeling to predict clinical outcomes have been reported only in the past six to seven years [27]. This is because of the time constraint that the simulation results synthesized with clinical data obtained at the beginning of the procedure should be completed during pulmonary vein isolation (less than an hour). It took more than 50 years to apply AF computational modeling in clinical medicine because it requires high computational power for sophisticated and complex simulations. However, with the innovative development of graphic processing units (GPUs) and parallel computing methods in the last 20 years, it is now possible to apply AF computational modeling to AF catheter ablation in real-time (Fig. 2) [28]. Table 1 summarizes the applications that are possible with cutting-edge AF computational modeling.

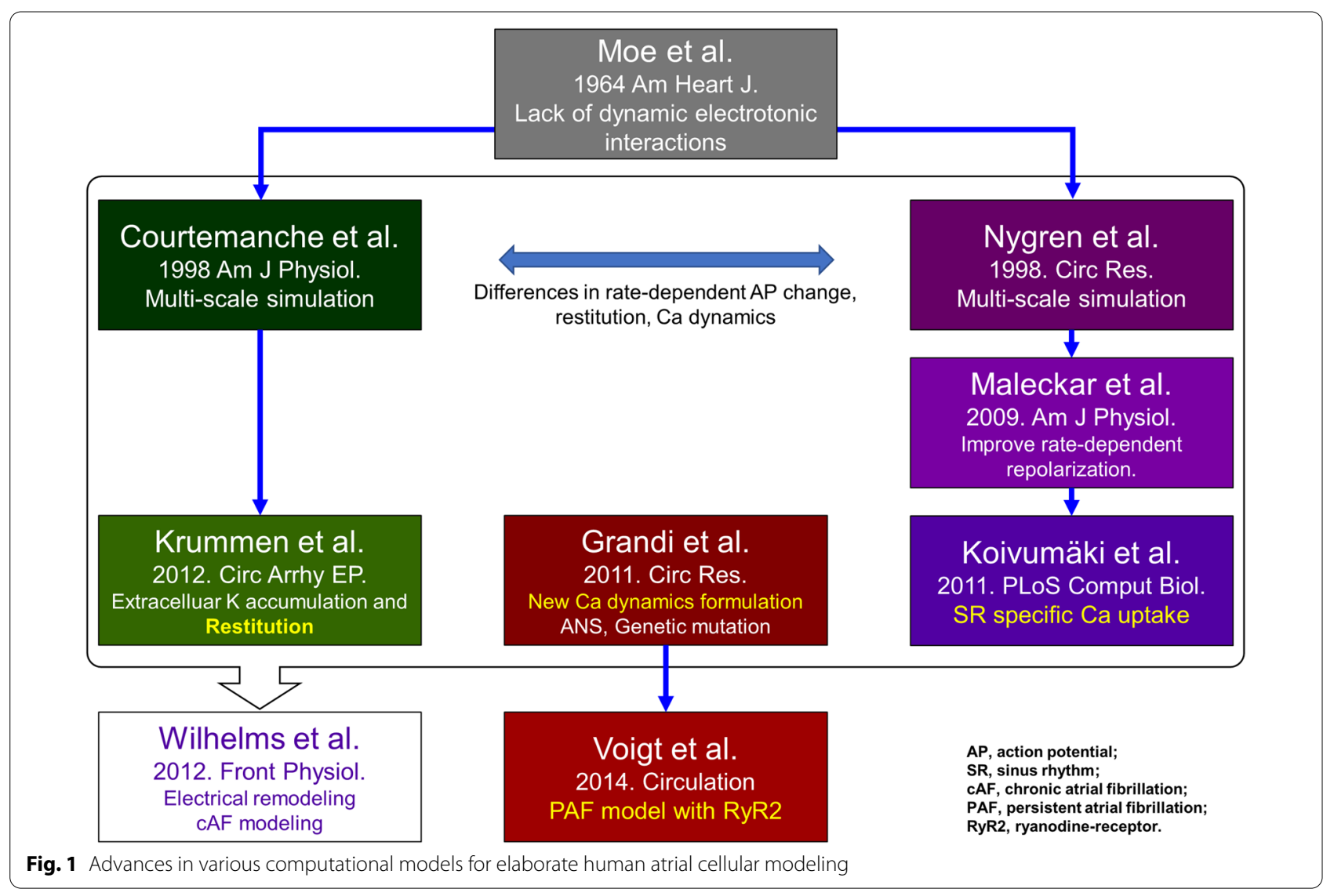




\section{GPU Based Simulation 80 fold faster Simulation Time}

KR2013-0037732; PCT/KR2013-008057 Kwan SS, Pak HN, et al. IEEE EMBC 2013;2013:1522-5.

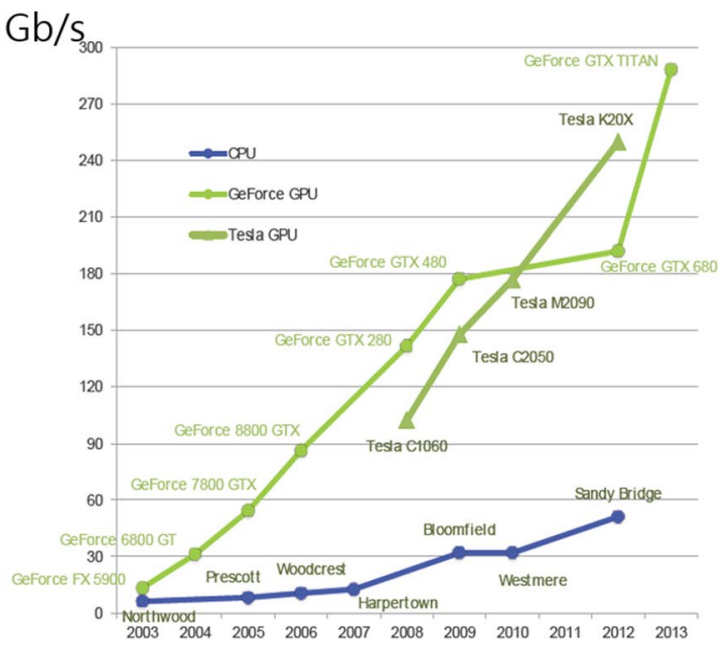

\section{Comparison to Others}

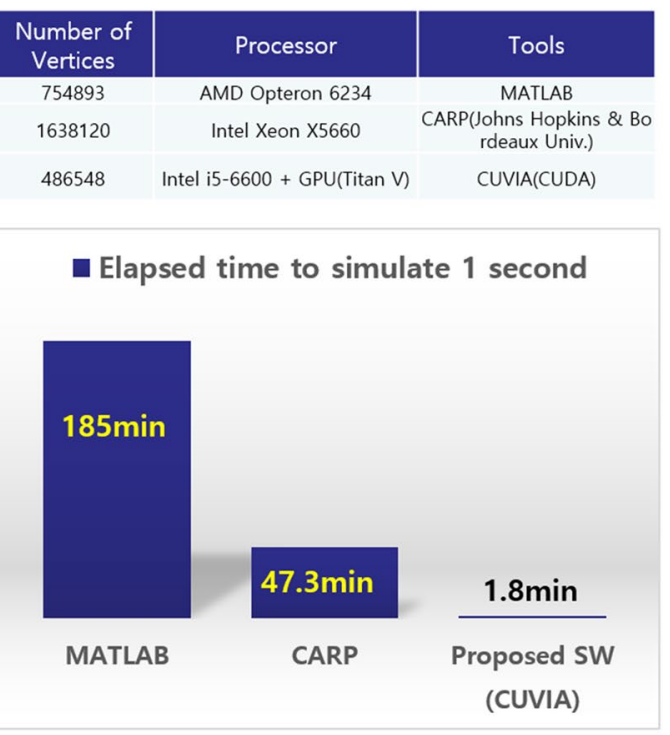

Fig. 2 Memory bandwidth growth rate of CPU and GPU, and performance comparison between software (SW) implemented with parallel computing and others SW. (Left) The finite difference method applied to AF simulation is particularly suitable for parallel computing due to its iterative nature and can solve partial differential equations at high speed through GPUs with high bandwidth and many cores compared to CPU. (Right) CUVIA SW, which is implemented based on CUDA-based parallel architecture, shows significant performance compared to CARP (Johns Hopkins \& Bordeaux Univ.) and MATLAB-based SW designed with serial architecture

Table 1 Applications of AF computational modeling

High-density map of entire chamber

Reproducible condition control

Virtual interventions, not possible clinically or experimentally

In-depth mechanistic research

Prediction of clinical outcomes

\section{Application of AF computational modeling 1: high-density map of entire chamber}

With the introduction of high-density mapping to clinical electrophysiology, the understanding of various tachyarrhythmia mechanisms has been improving [29]. High-density maps of decelerating zones and ablation gaps have enabled effective arrhythmia interventions with minimal ablation-induced tissue damage [30]. Nevertheless, clinical high-density mapping using contact electrograms has a fundamental limitation pertaining to sequential pointby-point mapping. The limited mapping field and the electrogram differences according to the electrode size or arrangement are weaknesses of multi-electrode catheter mapping that are difficult to overcome. Sequential contact maps work well in the pacing state or organized regular tachycardia, but the wave dynamics of disorganized AF cannot be implemented without an entire cardiac chamber map. On the other hand, if computational modeling is applied to a clinical voltage/activation map acquired during sinus rhythm, and then, virtual AF is induced by simulation, not only entire AF chamber mapping but also biatrial high-density AF modeling is possible [31, 32]. The example of qualitative comparison of the clinical and virtual local activation map and voltage map obtained during high RA pacing is shown in Fig. 3. Our research team uses AF computational modeling with a spatial resolution of $300 \mu \mathrm{m}$ and a temporal resolution of $0.1 \mathrm{~ms}$ [32]. 
A. NavX local activation and voltage maps. Clinical local activation map $(n=300-550)$

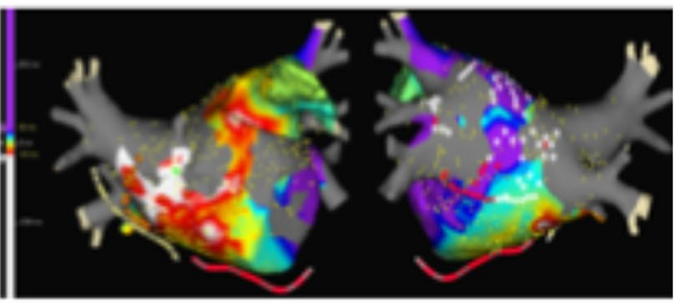

Clinical voltage map $(n=300-550)$

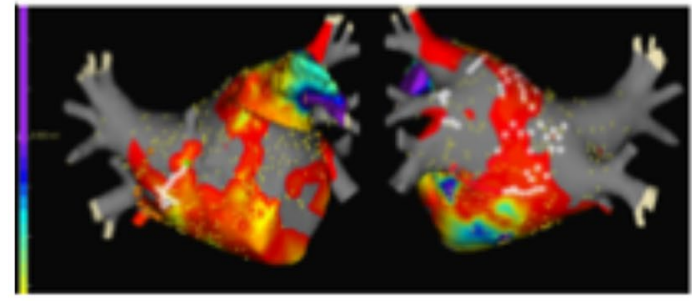

B. Virtual local activation and voltage map derived from clinical substrate map
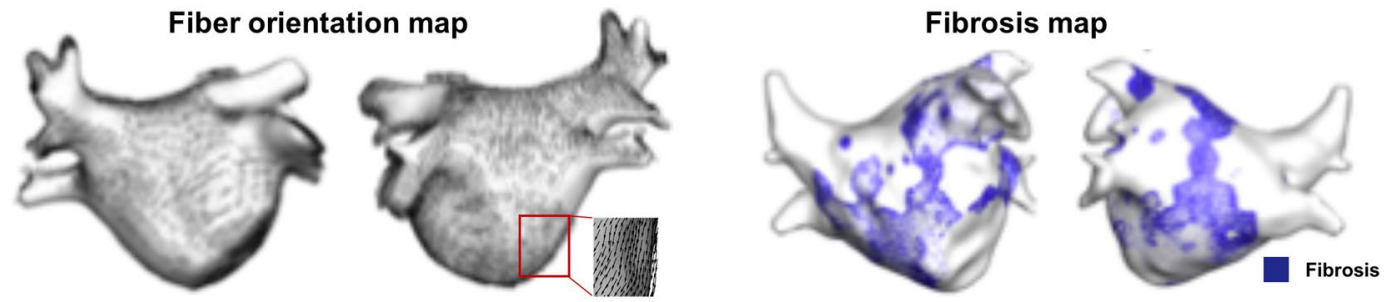

Virtual local activation map $(n=500,000)$
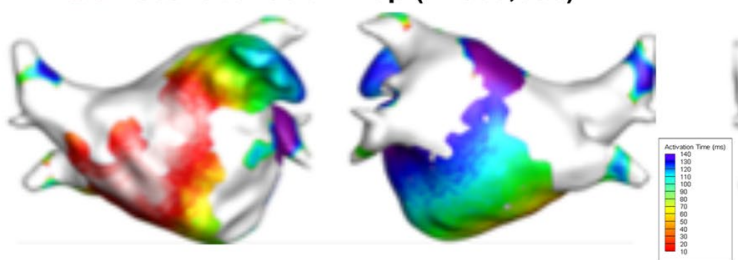

Virtual voltage map $(n=500,000)$
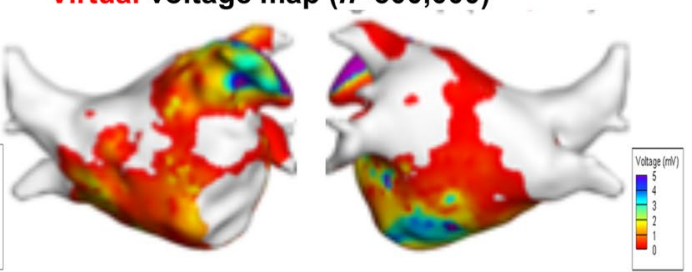

Fig. 3 High-resolution virtual local activation and voltage maps derived from clinical substrate maps are quantitatively compared with

low-resolution clinical local activation and voltage maps. A A clinical substrate map consisting of local activation and voltage maps was acquired by the NavX system. B Virtual local activation and voltage maps for virtual atrial remodeling are derived from clinical substrate maps

\section{Application of AF computational modeling 2: reproducible condition control}

Simulation-based entire chamber mapping of AF is a useful and unique method to understand and analyze AF wave dynamics [33, 34]. AF simulation can also be applied to detect extra-pulmonary vein (PV) AF drivers or rotors. Phase singularity points (PSs), dominant frequency (DF), Shannon's entropy (ShEn), and complex fractionated atrial electrogram (CFAE) have been used as indicators representing persistent rotational reentry or rotor [35-37]. Which of these indicators representing various AF wave-dynamics best reflects the AF rotor can be confirmed by targeted virtual ablation? However, virtual ablation should be performed under exactly the same conditions and same timing to evaluate their contributions to the AF maintenance mechanism. Hwang et al., using the characteristic of reproducible conditional control of AF computational modeling, ablated four different indicators under exactly the same conditions and timing and proved that $\mathrm{DF}$ is the predominant parameter of $\mathrm{AF}$ maintenance (Fig. 4A) [38].

\section{Application of computational modeling 3: virtual interventions, not possible experimentally or clinically}

In addition to reproducible condition control, tests under the same conditions that are not possible by clinical or experimental studies can be conducted through AF computational modeling. The AF termination or defragmentation rate after DF ablation was the highest among the AF wave-dynamics parameters. However, the position of the DF during the prolonged AF maintenance period was not stationary and a significant portion was meandering [33]. This DF spatiotemporal instability might have a negative effect on the DF ablation during AF. This virtual intervention can also be applied to evaluate the effectiveness of AADs under the same conditions [39].

Computational modeling can reproduce action potentials as well as virtual bipolar or unipolar electrograms according to the catheter-tissue contact angle and direction. Hwang et al. simulated three different types of multi-electrode catheters with different electrode sizes and arrangements and demonstrated the difference in the 


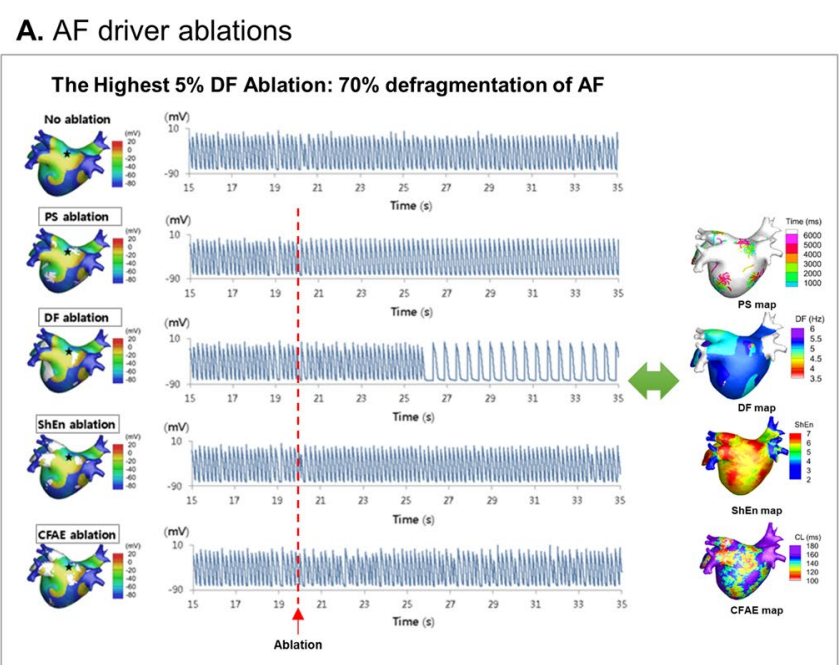

B. Virtual bipolar catheter voltage maps

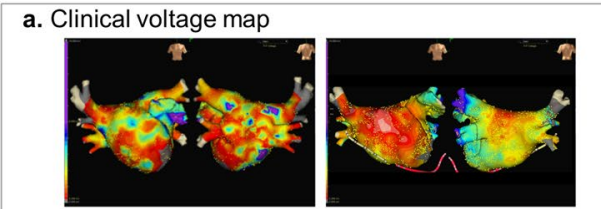

b. Virtual voltage map

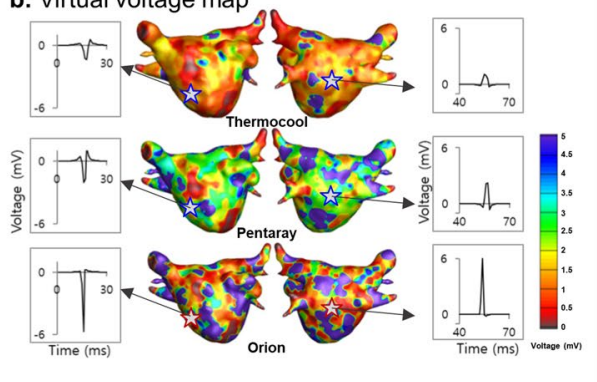

Fig. 4 Visualization of 3D voltage maps, action potential curves, and four different indicators for electroanatomical analysis-based virtual ablation based on the similarity of clinical versus virtual voltage maps. A Results of four different indicators analyzed in reproducible condition control for AF driver ablation. B Differences in the electrogram amplitudes simulated with three types of multi-electrode catheters with different electrode sizes and arrangements under the same clinical conditions

electrogram amplitude of the atrial voltage map obtained under exactly the same clinical conditions (Fig. 4B) [40].

\section{Application of computational modeling 4: in-depth mechanistic research}

The advantage of computational modeling is that various elements close to reality can be added to the basic AF model. From the simple modeling of a patient's cardiac CT image covered by a human atrial cell model [41], sophisticated AF modeling with a local degree of fibrosis, myocardial fiber orientation, and cardiac MRI-based scar character is possible by applying clinical atrial voltage/ local activation maps [31, 32, 42]. Nevertheless, studies reflecting extra-cardiac effects, especially cardiac autonomic nerve effects, are not easy to clinically or experimentally conduct. Hwang et al. added cardiac autonomic nerve to AF computational modeling according to an existing histology study; this created an environment for functional parasympathetic and sympathetic activation and demonstrated PV triggers by Phase 3 early after depolarization [43]. This suggests that it is possible to study the AF initiation mechanism with computational modeling and demonstrated the PV reentry and ganglionated plexi ablation effects after the PV trigger (Fig. 5).

\section{Application of computational modeling 5: prediction of clinical outcomes}

Despite its diverse applications in the research field of AF computational modeling, its clinical application has begun recently. Speed is a key factor in the clinical application of computational modeling, and this became possible with the improvement of computational power via the development of GPUs and parallel computing methods. Jacquemet et al. introduced five studies on computational modeling-guided AF ablation [27]. Among them, the calculation time of the CUVIA software (Laonmed Inc., Seoul, Korea) developed by our research team was the fastest, at 1.2 min per $1 \mathrm{~s}$ of AF simulation (Intel i5 6600+ GPU Titan V, 500,000 nodes). Therefore, we would like to introduce virtual AF ablation and virtual AAD using CUVIA-AF computational modeling.

\section{Computational modeling for AF ablation}

The first application of AF computational modeling to clinical practice was to determine the optimal lesion set for a surgical maze or catheter ablation [41, 44-47]. At this time, all of those studies were conducted as retrospective studies because lengthy computing time was required to evaluate $\mathrm{AF}$ intervention and termination using computational modeling [27]. Hwang et al. tested the effects of five different extra-PV linear or electrogram-guided lesion sets by mapping the data of 20 patients with persistent AF who underwent AF catheter ablation using CUVIA software [41]. In that study, the lesion set showing the best termination in the AF simulation showed high agreement with the clinically chosen most effective lesion set. Based on these results, Shim et al. conducted a prospective randomized clinical trial, CUVIA-AF I, which compared the effectiveness of 
A. Ganglionated plexi modeling
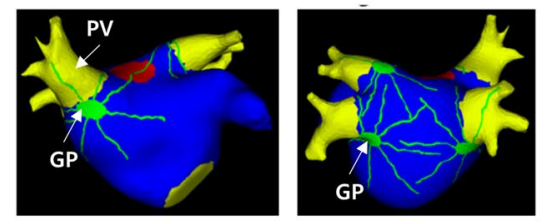

B. Pulmonary-vein Phase 3 early after-depolarization

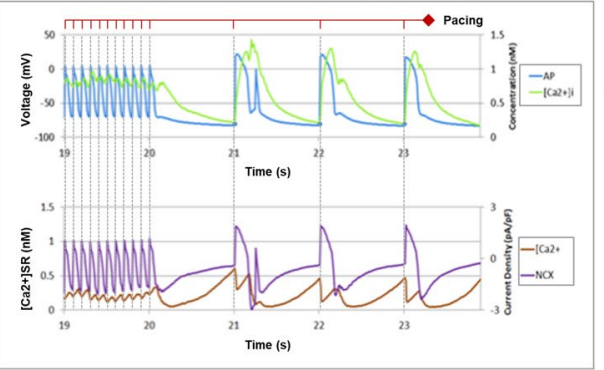

C. Pulmonary-vein automaticity

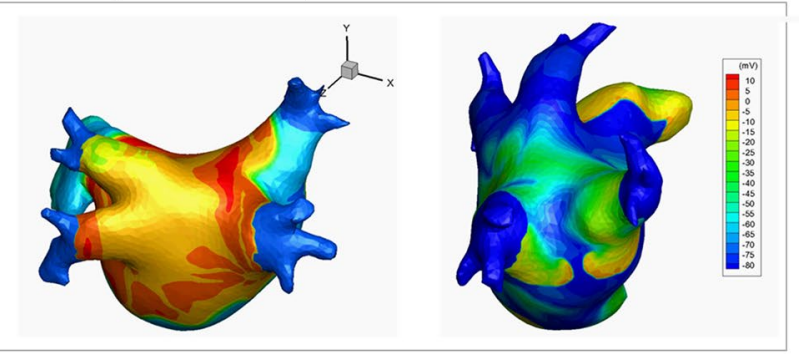

D. Circumferential pulmonary-vein isolation (including GP)

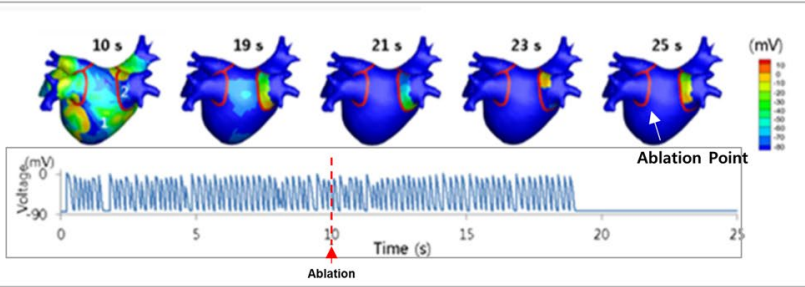

Fig. 5 Effect of PV reentry and ganglionated plexi ablation after PV triggers in Phase 3 early after depolarization via autonomic nerve modeling. AF computational modeling of cardiac autonomic nerve effects, which is difficult to perform clinically or experimentally, will enable further studies for AF initiation mechanisms. A An example of ganglionated plexi (GP) modeling. B Abnormal depolarization was observed after a change of pacing cycle length. C 3D action potential map showing pulmonary-vein automaticity in 3D left atrium model with GP. D AF was terminated after virtual circumferential pulmonary vein isolation including GP. Images were taken from [43]

the extra-PV ablation lesion set determined by computational modeling and the operator's experience with 108 patients with persistent AF (Fig. 6A) [48]. Kim et al. demonstrated that modeling-guided AF ablation was superior to empirical ablation rhythm outcomes after long-term follow-ups of this randomized clinical trial for more than 32 months [49]. Although CUVIA-AF
I was a prospective study, the cardiac CT data of each target patient were delivered to the core laboratory six hours before the procedure, and a simple human atrial cell model was applied to the left atrial anatomy. Boyle et al. published a computational modeling-guided rotor ablation as a proof of concept study targeting the rotational reentry around the atrial scar characterized by
A. The Outcome of CUVIAAF I
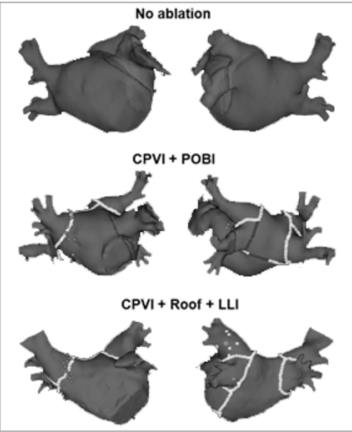

a

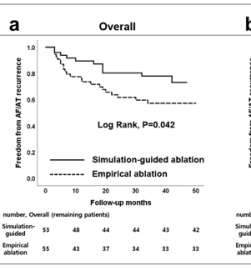

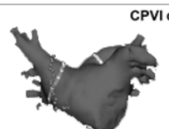

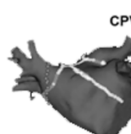

CPVI+ POBI + AL

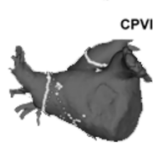

CPVI+CFAE

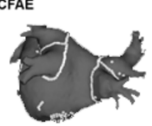

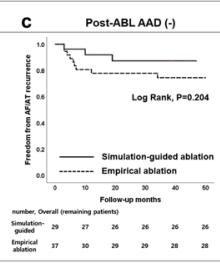

B. The Outcome of CUVIAAF II
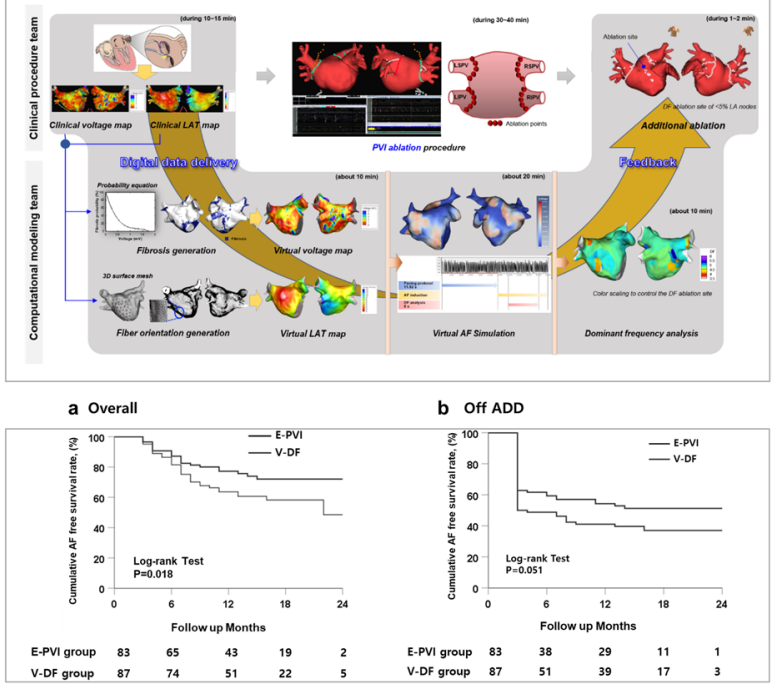

Fig. 6 Clinical trial outcomes of CUVIA-AF I and CUVIA-AF II using AF computational modeling. A CUVIA-AF I compared the effectiveness of the extra-PV ablation as determined by computational modeling and the operator's experience. Image was taken from [49]. B CUVIA-AF II evaluated the effect of additional DF ablation by AF-DF maps. Both clinical trials were analyzed during the clinical PV isolation procedure 
late gadolinium enhancement of cardiac MRI image [42].

After that, we upgraded to CUVIA version 3.0 to further improve the computational speed. This version is an AF simulation that reflects anatomy, histology, and electrophysiology by applying the endocardial voltage and local activation maps acquired at the beginning of the AF ablation procedure, enabling DF analysis within $40 \mathrm{~min}$ $[31,32]$. Accordingly, another randomized clinical trial, CUVIA-AF II, was conducted to compare and evaluate the effect of additional DF ablation by analyzing the onsite-acquired AF-DF map during the PV isolation procedure (Fig. 6B). CUVIA-AF II consistently confirmed the rhythm outcome of the virtual modeling-guided AF ablation was superior to empirical ablation in patients with persistent AF (data not shown). Although extra-PV foci are a major cause of recurrence in persistent AF, the appropriate target set is unclear with the current mapping technology. Therefore, computational modelingguided AF ablation will be an important breakthrough in targeting the appropriate extra-PV foci and improving the results of future procedures.

\section{Computational modeling for virtual antiarrhythmic drugs}

In the application of computational modeling, the virtual AAD test is much more complicated than virtual AF ablation, which tests the effect of a simple conduction block. Moreover, since patch-clamp studies verifying the various ion channel effects of AADs in human atrial cells are very limited, we needed to borrow the results of animal cellular experiments (Table 2). Hwang et al. conducted virtual AAD tests in realistic AF computational modeling that applied atrial anatomy, histology, and electrophysiology using the data of 20 patients who underwent endocardial voltage mapping at the time of $\mathrm{AF}$ catheter ablation [39]. Through computational modeling, they evaluated personalized electrophysiological effects and wave-dynamics changes after AAD administration in a dose-dependent manner and demonstrated the difference in AAD response according to the PITX2 genotype. Based on this proof of concept study, we are preparing another prospective randomized clinical trial, CUVIAAF III, which will compare and evaluate the effectiveness of virtual AAD-guided medical therapy.

\section{Current limitation and future perspectives}

The current AF computational modeling has several limitations, such as monolayer, image dependency, individual bias by segmentation, and overcoming of computation time for real-time. However, the machine learning-accelerated computational fluid dynamics technology recently introduced by Kochkov et al. [65] has potential for realtime AF modeling, and graph- and mesh-based deep learning technology can be expected to be applied as a cutting-edge diagnostic technology using the simulation results. As computational power continues to improve along with the development of artificial intelligence, the clinical application of AF computational modeling will be a popular diagnostic method in the future.

\section{Conclusions}

AF computational modeling will be utilized as an important breakthrough to improve the rhythm outcome of medical or interventional AF management. Various AF

Table 2 References for atrial cell ion currents depending on AADs

\begin{tabular}{|c|c|c|c|c|}
\hline AADs & Referencew & Animal/human model & Method & lon current change \\
\hline $\begin{array}{l}\text { Amiodarone } \\
(5 \mu \mathrm{M}, 10 \mu \mathrm{M})\end{array}$ & Varela et al. [50] & Canine atrial model & $\begin{array}{l}\text { Microelectrode recording and patch- } \\
\text { clamp }\end{array}$ & $\begin{array}{l}\text { gK1, gKur, gNa, gKr, gCaL, gKs } \\
\text { Ach }\end{array}$ \\
\hline $\begin{array}{l}\text { Sotalol } \\
(60 \mu \mathrm{M}, 10 \mathrm{mM})\end{array}$ & $\begin{array}{l}\text { Ducroq et al. [51] } \\
\text { Lin et al. [52] }\end{array}$ & $\begin{array}{l}\text { Rabbit/Human embryonic kidney cells } \\
\text { Xenopus oocytes }\end{array}$ & $\begin{array}{l}\text { Bipolar Ag electrode recoding and } \\
\text { patch clamp } \\
\text { Two-electrode voltage clamp }\end{array}$ & $\mathrm{gNa}, \mathrm{gKr}, \mathrm{gKs}$ \\
\hline $\begin{array}{l}\text { Dronedarone } \\
(3 \mu \mathrm{M}, 10 \mu \mathrm{M})\end{array}$ & $\begin{array}{l}\text { Chen et al. [53] } \\
\text { Gautier et al. [54] } \\
\text { Ji et al. [55] } \\
\text { Wegener et al. [56] }\end{array}$ & $\begin{array}{l}\text { Rat } \\
\text { Guinea pig ventricular cardiomyocyte } \\
\text { Dog ventricular myocytes } \\
\text { Guinea pig myocytes }\end{array}$ & $\begin{array}{l}\text { Whole-cell, perforated patch voltage- } \\
\text { clamp }\end{array}$ & gCaL, gKs, gNa, gK1, gKr, gCaL \\
\hline $\begin{array}{l}\text { Flecainide } \\
(5 \mu \mathrm{M}, 15 \mu \mathrm{M})\end{array}$ & $\begin{array}{l}\text { Geng et al. [57] } \\
\text { Yue et al. [58] } \\
\text { Wang et al. [59] } \\
\text { Hilliard et al. [60] }\end{array}$ & $\begin{array}{l}\text { Human pluripotent stem cell-derived } \\
\text { ventricular cardiomyocyte } \\
\text { Human right atrial appendage } \\
\text { Human pluripotent stem cell-derived } \\
\text { ventricular cardiomyocyte } \\
\text { Canine, murine ventricular model }\end{array}$ & $\begin{array}{l}\text { Whole-cell patch voltage clamp, micro- } \\
\text { scope, and confocal laser-scanning unit }\end{array}$ & gNa, gKur, gNa, gto, gCaL \\
\hline $\begin{array}{l}\text { Propafenone } \\
(5 \mu \mathrm{M}, 10 \mu \mathrm{M})\end{array}$ & $\begin{array}{l}\text { Wang et al. [61] } \\
\text { Paul et al. [62] } \\
\text { Seki et al. [63] } \\
\text { Delgado et al. [64] }\end{array}$ & $\begin{array}{l}\text { Human Embryonic kidney cells } \\
\text { Human atrial myocytes } \\
\text { Guinea pig ventricular myocytes }\end{array}$ & Whole-cell patch voltage clamp & gNa, gto, gCaL, gKur, gKr, \\
\hline
\end{tabular}


simulation models are now just around the corner for clinical applications.

\begin{abstract}
Abbreviations
AF: Atrial fibrillation; AADs: Antiarrhythmic drugs; AFCA: Atrial fibrillation catheter ablation; MRI: Magnetic resonance imaging; GPUs: Graphic processing units; PV: Pulmonary vein; PS: Phase singularity; DF: Dominant frequency; ShEn: Shannon's entropy; CFAE: Complex fractionated atrial electrogram; CT: Computed tomography.
\end{abstract}

\section{Acknowledgements}

We thank Mr. John Martin for his linguistic assistance.

\section{Authors' contributions}

O.S.K. and H.N.P wrote the manuscript. I.H. contributed to the collection of reference materials. All authors read and approved the final manuscript.

\section{Funding}

This work was supported by a grant [HI19C0114] and [HI21C0011] from the Ministry of Health and Welfare and a grant [NRF-2020R1A2B01001695] from the Basic Science Research Program run by the National Research Foundation of Korea (NRF) which is funded by the Ministry of Science, ICT \& Future Planning (MSIP)

\section{Availability of data and materials}

Data are available from the corresponding author on reasonable request due to privacy or other restrictions.

\section{Declarations}

\section{Ethics approval and consent to participate}

Not applicable.

\section{Consent for publication}

Not applicable.

\section{Competing interests}

The authors declare that they have no competing interests.

Received: 21 July 2021 Accepted: 28 September 2021

Published online: 15 December 2021

\section{References}

1. Kim D, Yang PS, Jang E, Yu HT, Kim TH, Uhm JS, et al. 10-year nationwide trends of the incidence, prevalence, and adverse outcomes of nonvalvular atrial fibrillation nationwide health insurance data covering the entire Korean population. Am Heart J. 2018;202:20-6.

2. Steg PG, Alam S, Chiang CE, Gamra H, Goethals M, Inoue H, et al. Symptoms, functional status and quality of life in patients with controlled and uncontrolled atrial fibrillation: data from the RealiseAF cross-sectional international registry. Heart (British Cardiac Society). 2012;98(3):195-201.

3. Healey JS, Connolly SJ, Gold MR, Israel CW, Van Gelder IC, Capucci A, et al. Subclinical atrial fibrillation and the risk of stroke. N Engl J Med. 2012;366(2):120-9.

4. Lee JY, Kim TH, Yang PS, Lim HE, Choi EK, Shim J, et al. Korean atrial fibrillation network genome-wide association study for early-onset atrial fibrillation identifies novel susceptibility loci. Eur Heart J. 2017;38(34):2586-94.

5. Parvez B, Vaglio J, Rowan S, Muhammad R, Kucera G, Stubblefield T, et al. Symptomatic response to antiarrhythmic drug therapy is modulated by a common single nucleotide polymorphism in atrial fibrillation. J Am Coll Cardiol. 2012;60(6):539-45.

6. Preliminary report: effect of encainide and flecainide on mortality in a randomized trial of arrhythmia suppression after myocardial infarction. New Engl J Med. 1989;321(6):406-12.
7. Waldo AL, Camm AJ, deRuyter H, Friedman PL, MacNeil DJ, Pauls JF, et al. Effect of d-sotalol on mortality in patients with left ventricular dysfunction after recent and remote myocardial infarction. The SWORD Investigators. Survival With Oral d-Sotalol. Lancet (London, England). 1996:348(9019):7-12

8. Haïssaguerre M, Jaïs P, Shah DC, Takahashi A, Hocini M, Quiniou G, et al. Spontaneous initiation of atrial fibrillation by ectopic beats originating in the pulmonary veins. N Engl J Med. 1998;339(10):659-66.

9. Cox JL, Schuessler RB, D'Agostino HJ Jr, Stone CM, Chang BC, Cain $M E$, et al. The surgical treatment of atrial fibrillation. III. Development of a definitive surgical procedure. J Thorac Cardiovasc Surg. 1991;101(4):569-83.

10. Marrouche NF, Brachmann J, Andresen D, Siebels J, Boersma L, Jordaens $L$, et al. Catheter ablation for atrial fibrillation with heart failure. N Engl J Med. 2018;378(5):417-27.

11. Packer DL, Mark DB, Robb RA, Monahan KH, Bahnson TD, Poole JE, et al. Effect of catheter ablation vs antiarrhythmic drug therapy on mortality, stroke, bleeding, and cardiac arrest among patients with atrial fibrillation: the CABANA randomized clinical trial. JAMA. 2019;321(13):1261-74.

12. Kim M, Yu HT, Kim J, Kim TH, Uhm JS, Joung B, et al. Atrial fibrillation and the risk of ischaemic strokes or intracranial haemorrhages: comparisons of the catheter ablation, medical therapy, and non-atrial fibrillation population. Europace. 2021;23(4):529-38.

13. Kim D, Yang PS, Sung JH, Jang E, Yu HT, Kim TH, et al. Less dementia after catheter ablation for atrial fibrillation: a nationwide cohort study. Eur Heart J. 2020;41(47):4483-93.

14. Park JW, Yang PS, Bae HJ, Yang SY, Yu HT, Kim TH, et al. Five-year change in the renal function after catheter ablation of atrial fibrillation. J Am Heart Assoc. 2019:8(17):e013204.

15. Park JW, Yu HT, Kim TH, Uhm JS, Kim JY, Joung B, et al. Trends and outcome of catheter ablation of atrial fibrillation over 9 years- focus on empirical extra-pulmonary vein ablation. Circ J. 2019;83(2):304-12.

16. Moe GK, Rheinboldt WC, Abildskov JA. A computer model of atrial fibrillation. Am Heart J. 1964;67:200-20.

17. Trayanova NA. Mathematical approaches to understanding and imaging atrial fibrillation: significance for mechanisms and management. Circ Res. 2014;114(9):1516-31.

18. Courtemanche M, Ramirez RJ, Nattel S. Ionic mechanisms underlying human atrial action potential properties: insights from a mathematical model. Am J Physiol. 1998;275(1):H301-21.

19. Nygren A, Fiset C, Firek L, Clark JW, Lindblad DS, Clark RB, et al. Mathematical model of an adult human atrial cell: the role of $\mathrm{K}+$ currents in repolarization. Circ Res. 1998;82(1):63-81.

20. Wilhelms M, Hettmann H, Maleckar MM, Koivumäki JT, Dössel O, Seemann G. Benchmarking electrophysiological models of human atrial myocytes. Front Physiol. 2012;3:487.

21. Krummen DE, Bayer JD, Ho J, Ho G, Smetak MR, Clopton P, et al. Mechanisms of human atrial fibrillation initiation: clinical and computational studies of repolarization restitution and activation latency. Circ Arrhythm Electrophysiol. 2012;5(6):1149-59.

22. Maleckar MM, Greenstein JL, Giles WR, Trayanova NA. K+ current changes account for the rate dependence of the action potential in the human atrial myocyte. Am J Physiol Heart Circ Physiol. 2009;297(4):H1398-410.

23. Koivumäki JT, Korhonen T, Tavi P. Impact of sarcoplasmic reticulum calcium release on calcium dynamics and action potential morphology in human atrial myocytes: a computational study. PLoS Comput Biol. 2011;7(1):e1001067.

24. Voigt N, Heijman J, Wang Q, Chiang DY, Li N, Karck M, et al. Cellular and molecular mechanisms of atrial arrhythmogenesis in patients with paroxysmal atrial fibrillation. Circulation. 2014;129(2):145-56.

25. McDowell KS, Vadakkumpadan F, Blake R, Blauer J, Plank G, MacLeod RS, et al. Methodology for patient-specific modeling of atrial fibrosis as a substrate for atrial fibrillation. J Electrocardiol. 2012;45(6):640-5.

26. Aslanidi OV, Colman MA, Stott J, Dobrzynski H, Boyett MR, Holden AV, et al. 3D virtual human atria: A computational platform for studying clinical atrial fibrillation. Prog Biophys Mol Biol. 2011;107(1):156-68.

27. Jacquemet $\mathrm{V}$. Lessons from computer simulations of ablation of atrial fibrillation. J Physiol. 2016;594(9):2417-30.

28. Kwon SS, Yun YH, Hong SB, Pak HN, Shim EB. A patient-specific model of virtual ablation for atrial fibrillation. Annu Int Conf IEEE Eng Med Biol Soc. 2013:2013:1522-5. 
29. Aziz Z, Shatz D, Raiman M, Upadhyay GA, Beaser AD, Besser SA, et al. Targeted ablation of ventricular tachycardia guided by wavefront discontinuities during sinus rhythm: a new functional substrate mapping strategy. Circulation. 2019;140(17):1383-97.

30. Tung R, Raiman M, Liao H, Zhan X, Chung FP, Nagel R, et al. Simultaneous endocardial and epicardial delineation of $3 \mathrm{D}$ reentrant ventricular tachycardia. J Am Coll Cardiol. 2020;75(8):884-97.

31. Lim B, Kim J, Hwang M, Song JS, Lee JK, Yu HT, et al. In situ procedure for high-efficiency computational modeling of atrial fibrillation reflecting personal anatomy, fiber orientation, fibrosis, and electrophysiology. Sci Rep. 2020;10(1):2417.

32. Lim B, Park JW, Hwang M, Ryu AJ, Kim IS, Yu HT, et al. Electrophysiological significance of the interatrial conduction including cavo-tricuspid isthmus during atrial fibrillation. J Physiol. 2020;598(17):3597-612.

33. Li C, Lim B, Hwang M, Song JS, Lee YS, Joung B, et al. The spatiotemporal stability of dominant frequency sites in in-silico modeling of 3-dimensional left atrial mapping of atrial fibrillation. PLOS ONE. 2016;11(7):e0160017.

34. Lim B, Hwang M, Song JS, Ryu AJ, Joung B, Shim EB, et al. Effectiveness of atrial fibrillation rotor ablation is dependent on conduction velocity: an in-silico 3-dimensional modeling study. PLoS ONE. 2017;12(12):e0190398.

35. Pandit SV, Berenfeld O, Anumonwo JM, Zaritski RM, Kneller J, Nattel $\mathrm{S}$, et al. lonic determinants of functional reentry in a 2-D model of human atrial cells during simulated chronic atrial fibrillation. Biophys J. 2005;88(6):3806-21.

36. Ganesan AN, Kuklik P, Lau DH, Brooks AG, Baumert M, Lim WW, et al. Bipolar electrogram shannon entropy at sites of rotational activation: implications for ablation of atrial fibrillation. Circ Arrhythm Electrophysiol. 2013:6(1):48-57.

37. Nademanee K, McKenzie J, Kosar E, Schwab M, Sunsaneewitayakul B, Vasavakul T, et al. A new approach for catheter ablation of atrial fibrillation: mapping of the electrophysiologic substrate. J Am Coll Cardiol. 2004:43(11):2044-53.

38. Hwang M, Song JS, Lee YS, Li C, Shim EB, Pak HN. Electrophysiological rotor ablation in in-silico modeling of atrial fibrillation: comparisons with dominant frequency, shannon entropy, and phase singularity. PLoS ONE. 2016;11(2):e0149695.

39. Hwang I, Park JW, Kwon OS, Lim B, Hong M, Kim M, et al. Computational modeling for antiarrhythmic drugs for atrial fibrillation according to genotype. Front Physiol. 2021;12:650449.

40. Hwang M, Kim J, Lim B, Song JS, Joung B, Shim EB, et al. Multiple factors influence the morphology of the bipolar electrogram: an in silico modeling study. PLoS Comput Biol. 2019;15(4):6765

41. Hwang M, Kwon SS, Wi J, Park M, Lee HS, Park JS, et al. Virtual ablation for atrial fibrillation in personalized in-silico three-dimensional left atrial modeling: comparison with clinical catheter ablation. Prog Biophys Mol Biol. 2014;116(1):40-7.

42. Boyle PM, Zghaib T, Zahid S, Ali RL, Deng D, Franceschi WH, et al. Computationally guided personalized targeted ablation of persistent atrial fibrillation. Nat Biomed Eng. 2019;3(11):870-9.

43. Hwang M, Lim B, Song JS, Yu HT, Ryu AJ, Lee YS, et al. Ganglionated plexi stimulation induces pulmonary vein triggers and promotes atrial arrhythmogenecity: in silico modeling study. PLOS ONE. 2017;12(2):e0172931.

44. Trayanova NA, Boyle PM, Nikolov PP. Personalized imaging and modeling strategies for arrhythmia prevention and therapy. Curr Opin Biomed Eng. 2018;5:21-8.

45. Roney CH, Williams SE, Cochet H, Mukherjee RK, O'Neill L, Sim I, et al. Patient-specific simulations predict efficacy of ablation of interatrial connections for treatment of persistent atrial fibrillation. Europace. 2018;20(suppl_3):iii55-68.

46. Jacquemet V. Modeling left and right atrial contributions to the ECG: a dipole-current source approach. Comput Biol Med. 2015;65:192-9.

47. Loewe A, Krueger MW, Holmqvist F, Dössel O, Seemann G, Platonov PG. Influence of the earliest right atrial activation site and its proximity to interatrial connections on P-wave morphology. Europace. 2016;18(suppl 4):iv35-43.
48. Shim J, Hwang M, Song JS, Lim B, Kim TH, Joung B, et al. Virtual in-silico modeling guided catheter ablation predicts effective linear ablation lesion set for longstanding persistent atrial fibrillation: multicenter prospective randomized study. Front Physiol. 2017:8:792.

49. Kim IS, Lim B, Shim J, Hwang M, Yu HT, Kim TH, et al. Clinical usefulness of computational modeling-guided persistent atrial fibrillation ablation: updated outcome of multicenter randomized study. Front Physiol. 2019;10:1512

50. Varela M, Colman MA, Hancox JC. Atrial heterogeneity generates re-entrant substrate during atrial fibrillation and anti-arrhythmic drug action: mechanistic insights from canine atrial models. PLoS Comput Biol. 2016;12(12):5245.

51. Ducroq J, Printemps R, Guilbot S, Gardette J, Salvetat C, Le Grand MJ, et al. Action potential experiments complete hERG assay and QT-interval measurements in cardiac preclinical studies. J Pharmacol Toxicol Methods. 2007:56(2):159-70.

52. Lin C, Ke X, Cvetanovic I, Ranade V, Somberg JJC. The effect of high extracellular potassium on IKr inhibition by anti-arrhythmic agents. Cardiology. 2007;108(1):18-27.

53. Chen $K-H$, Xu X-H, Sun H-Y, Du X-L, Liu H, Yang L, et al. Distinctive property and pharmacology of voltage-gated sodium current in rat atrial vs ventricular myocytes. Heart Rhythm. 2016;13(3):762-70.

54. Gautier P, Guillemare E, Marion A, Bertrand J-P, Tourneur Y, Nisato DJ. Electrophysiologic characterization of dronedarone in guinea pig ventricular cells. J Cardiovasc Pharmacol. 2003:41(2):191-202.

55. Ji Y, Varkevisser R, Opacic D, Bossu A, Kuiper M, Beekman JDM, et al. The inward rectifier current inhibitor PA-6 terminates atrial fibrillation and does not cause ventricular arrhythmias in goat and dog models. $\mathrm{Br} J$ Pharmacol. 2017;174(15):2576-90.

56. Wegener JW, Schulla V, Koller A, Klugbauer N, Feil R, Hofmann F. Control of intestinal motility by the $\mathrm{Ca}(\mathrm{v}) 1.2 \mathrm{~L}$-type calcium channel in mice. FASEB J. 2006;20(8):1260-2.

57. Geng L, Kong C-W, Wong AO, Shum AM-Y, Chow MZ, Che H, et al. Probing flecainide block of INa using human pluripotent stem cell-derived ventricular cardiomyocytes adapted to automated patch-clamping and 2D monolayers. FASEB J. 2018;294:61-72.

58. Yue L, Feng JL, Wang Z, Nattel S. Effects of ambasilide, quinidine, flecainide and verapamil on ultra-rapid delayed rectifier potassium currents in canine atrial myocytes. Cardiovasc Res. 2000;46(1):151-61.

59. Wang Z, Fermini B, Nattel S. Sustained depolarization-induced outward current in human atrial myocytes. Evidence for a novel delayed rectifier $\mathrm{K}+$ current similar to Kv1. 5 cloned channel currents. Circ Res. 1993;73(6):1061-76

60. Hilliard FA, Steele DS, Laver D, Yang Z, Le Marchand SJ, Chopra N, et al. Flecainide inhibits arrhythmogenic Ca2 + waves by open state block of ryanodine receptor $\mathrm{Ca} 2+$ release channels and reduction of $\mathrm{Ca} 2+$ spark mass. J Mol Cell Cardiol. 2010;48(2):293-301.

61. Wang GK, Edrich T, Wang S-Y. Time-dependent block and resurgent tail currents induced by mouse $34154-167$ peptide in cardiac Na+ channels. J Gen Physiol. 2006;127(3):277-89.

62. Paul AA, Witchel HJ, Hancox JC. Inhibition of the current of heterologously expressed HERG potassium channels by flecainide and comparison with quinidine, propafenone and lignocaine. $\mathrm{Br} J$ pharmacol. 2002;136(5):717-29.

63. Seki A, Motoya K, Watanabe S, Kubo I. Novel sensors for potassium, calcium and magnesium ions based on a silicon transducer as a lightaddressable potentiometric sensor. Anal Chim Acta. 1999;382(1-2):131-6.

64. Delgado C, Tamargo J, Henzel D, Lorente P. Effects of propafenone on calcium current in guinea-pig ventricular myocytes. Br J Pharmacol. 1993;108(3):721-7.

65. Kochkov D, Smith JA, Alieva A, Wang Q, Brenner MP, Hoyer S. Machine learning-accelerated computational fluid dynamics. Proc Natl Acad Sci USA. 2021;118(21):e2101784118. 